\title{
Efeito dos probióticos na prevenção e tratamento de câncer e Diabetes mellitus
}

\section{Effect of probiotics on the prevention and treatment of cancer and Diabetes mellitus}

Efecto de los probióticos en la prevención y tratamiento del cáncer y la Diabetes mellitus

Brena Lopes Miranda

ORCID: https://orcid.org/0000-0002-5083-1465 Universidade Federal do Piauí, Brasil

E-mail: b-rena13@hotmail.com

Diêgo de Oliveira Lima

ORCID:https://orcid.org/0000-0001-8211-9416

Universidade Federal do Piauí, Brasil

E-mail: di.oliveiralima@hotmail.com

Thalia Barbosa

ORCID:https://orcid.org/0000-0002-9285-7460

Universidade Federal do Piauí, Brasil

E-mail: thaliatauany20@gmail.com

Maria Lúcia de Moura Rocha Barbosa

ORCID: https://orcid.org/0000-0002-7633-9815 Universidade Federal do Piauí, Brasil

E-mail: mlesperanca@gmail.com

Higo José Neri da Silva

ORCID: https://orcid.org/0000-0003-2089-4147 Universidade Federal do Piauí, Brasil

E-mail: higoneri@gmail.com

Edite Maria Morais do Nascimento

ORCID: https://orcid.org/0000-0002-6519-688X Universidade Federal do Piauí, Brasil E-mail: editemaria214@gmail.com

Dorothy Emanuelly Acácio Vasconcellos Meira ORCID: https://orcid.org/0000-0001-6396-0453 Universidade Federal do Piauí, Brasil

E-mail:dorothyemanuelly864@gmail.com

Rute Emanuela da Rocha ORCID: https://orcid.org/0000-0002-3763-6665 Universidade Federal do Piaú, Brasil

E-mail: r.emanuelarochanutri@ufpi.edu.br

Kananda Pereira Almeida

ORCID: https://orcid.org/0000-0001-5735-5353 Universidade Federal do Piauí, Brasil

E-mail: kananda.almeida@hotmail.com

Tiago Soares

ORCID: https://orcid.org/0000-0003-4597-2607 Universidade Federal do Piaú, Brasil

E-mail: tiagosoaresnutricao@ufpi.edu.br

Cláudio Rivelino Freire Passos ORCID: https://orcid.org/0000-0002-1879-6217 Tecnólogo em Radiologia, Brasil

E-mail: claudiopassos1@hotmail.com

Thalyta Pereira Oliveria

ORCID: https://orcid.org/0000-0003-1213-2054 Universidade Federal do Piauí, Brasil

E-mail: thalyta.qui@hotmail.com

Antony Charles Oliveira do Nascimento

ORCID: https://orcid.org/0000-0003-1862-9328

Tecnólogo em Radiologia, Brasil

E-mail:charlesnascimento.the@hotmail.com

Keylla da Conceição Machado

ORCID: https://orcid.org/0000-0002-4335-2829 Universidade Federal do Piauí, Brasil

E-mail: keyllamachado06@hotmail.com

Amanda Miranda da Silva

ORCID: https://orcid.org/0000-0001-6476-4809 Universidade Federal do Piauí, Brasil E-mail: miraanda.sa@gmail.com 


\title{
Resumo
}

Dieta rica em probióticos ajuda na prevenção e tratamento de doenças, bem como no equilíbrio da microbiota intestinal. Esta é uma revisão bibliográfica que buscou avaliar o efeito dos probióticos na prevenção e tratamento de câncer e diabetes. As bases de dados eletrônicas foram: Scielo, LILACS, Biblioteca Virtual em Saúde, Google Acadêmico, Medline e Pubmed, artigos em inglês, espanhol e português, descritores: Probióticos/Probiotics, Probióticos e diabetes/Probiotics and diabetes e Probióticos e câncer/Probiotics and câncer; critérios de inclusão: artigos completos publicados na íntegra, disponíveis nos idiomas inglês, espanhol e português recorte temporal nos últimos 20 anos. Inicialmente encontrado 12.000 artigos com o descritor probióticos, demais descritores, foi obtido o resultado final de 210 artigos. Observou se que o câncer e a diabetes são doenças crônicas, consideradas pela Organização Mundial da Saúde (OMS) como problemas prioritários de saúde pública global, estudos têm demonstrado ações benéficas com o uso de probióticos com pesquisas in vivo e in vitro, no câncer, os probióticos reduzem os efeitos mutagênicos e genotóxicos, agem com três mecanismos de proteção ao hospedeiro, na diabetes, os probióticos podem melhorar as alterações metabólicas como resistência à insulina, hiperglicemia, inflamação, dislipidemia ou DHGNA em animais e agem com mecanismos benéficos. A utilização de probióticos tem um papel benéfico no tratamento de doenças crônicas não transmissíveis como câncer e diabetes. No câncer, as bactérias probióticas reduzem os efeitos mutagênicos e genotóxicos, os benefícios dos probióticos na diabetes mellitus são a melhora nas alterações metabólicas associadas à diabetes, como resistência à insulina, hiperglicemia e inflamação.

Palavras-chave: Probióticos; Câncer; Diabetes Mellitus.

\begin{abstract}
Diet rich in probiotics helps in the prevention and treatment of diseases, as well as in the balance of the intestinal microbiota. This is a literature review that sought to assess the effect of probiotics on the prevention and treatment of cancer and diabetes. The electronic databases were: Scielo, LILACS, Virtual Health Library, Google Scholar, Medline and Pubmed, articles in English, Spanish and Portuguese, descriptors: Probiotics / Probiotics, Probiotics and diabetes / Probiotics and diabetes and Probiotics and cancer / Probiotics and cancer; Inclusion criteria: full articles published in full, available in English, Spanish and Portuguese, in the last 20 years. Initially found 12,000 articles with the descriptor probiotics, other descriptors, the final result of 210 articles was obtained. It was observed that cancer and diabetes are chronic diseases, considered by the World Health Organization (WHO) as priority problems of global public health, studies have shown beneficial actions with the use of probiotics with in vivo and in vitro research in cancer, probiotics reduce mutagenic and genotoxic effects, act with three host protection mechanisms, in diabetes, probiotics can improve metabolic changes such as insulin resistance, hyperglycemia, inflammation, dyslipidemia or NAFLD in animals and act with beneficial mechanisms. The use of probiotics has a beneficial role in the treatment of chronic noncommunicable diseases such as cancer and diabetes. In cancer, probiotic bacteria reduce mutagenic and genotoxic effects, the benefits of probiotics in diabetes mellitus are the improvement in metabolic changes associated with diabetes, such as insulin resistance, hyperglycemia and inflammation.
\end{abstract}

Keywords: Probiotics; Cancer; Diabetes Mellitus.

\section{Resumen}

Una dieta rica en probióticos ayuda en la prevención y tratamiento de enfermedades, así como en el equilibrio de la microbiota intestinal. Esta es una revisión de la literatura que buscaba evaluar el efecto de los probióticos en la prevención y el tratamiento del cáncer y la diabetes. Las bases de datos electrónicas fueron: Scielo, LILACS, Virtual Health Library, Google Scholar, Medline y Pubmed, artículos en inglés, español y portugués, descriptores: Probióticos / Probióticos, Probióticos y diabetes / Probióticos y diabetes y Probióticos y cáncer / Probióticos y cáncer; Criterios de inclusión: artículos completos publicados íntegramente, disponibles en inglés, español y portugués, en los últimos 20 años. Inicialmente se encontraron 12.000 artículos con el descriptor probióticos, otros descriptores, se obtuvo el resultado final de 210 artículos. Se observó que el cáncer y la diabetes son enfermedades crónicas, consideradas por la Organización Mundial de la Salud (OMS) como problemas prioritarios de la salud pública global, estudios han demostrado acciones beneficiosas con el uso de probióticos con investigaciones in vivo e in vitro en cáncer. , los probióticos reducen los efectos mutagénicos y genotóxicos, actúan con tres mecanismos de protección del huésped, en la diabetes, los probióticos pueden mejorar cambios metabólicos como la resistencia a la insulina, hiperglucemia, inflamación, dislipidemia o NAFLD en animales y actúan con mecanismos beneficiosos. El uso de probióticos tiene un papel beneficioso en el tratamiento de enfermedades crónicas no transmisibles como el cáncer y la diabetes. En el cáncer, las bacterias probióticas reducen los efectos mutagénicos y genotóxicos, los beneficios de los probióticos en la diabetes mellitus son la mejora de los cambios metabólicos asociados con la diabetes, como la resistencia a la insulina, la hiperglucemia y la inflamación.

Palabras clave: Probióticos; Cáncer; Diabetes Mellitus. 


\section{Introdução}

Para a promoção e a proteção da saúde, a alimentação e nutrição são consideradas requisitos básicos, dado que elas possibilitam a afirmação do potencial de crescimento e desenvolvimento humano com qualidade de vida (Brasil, 2013). Nos últimos anos a substituição de uma alimentação caseira e natural por alimentos industrializados, tendo afetando a população em geral influenciando negativamente o processo de saúde/doença (Toloni et al., 2011).

É importante elucidar que uma alimentação saudável deve conter alimentos funcionais, ricos em nutrientes em quantidades adequadas e ser de fácil acesso, atrativa e agradável ao paladar. De modo que os profissionais nutricionistas compreendem que para o planejamento adequado das dietas, vale atender ao habito alimentar, contemplar as preferencias, as aversões, e serem consideradas a cultura, a nacionalidade, as questões religiosas e socioeconômicas (Martins, Walder \& Rubiatti, 2010).

Outro ponto relevante a considerar é que a Secretaria de Vigilância Sanitária do Ministério da Saúde (ANVISA) no ano de 1999 regulamentou o termo Alimento Funcional “[...] como um alimento ou ingrediente que além das funções nutricionais básicas, quando consumido como parte da dieta usual, produz efeitos metabólicos e/ou efeitos benéficos à saúde, devendo ser seguro para consumo sem supervisão médica" (ANVISA, 1999). Essa regulamentação é de fundamental importância, pois a demanda por produtos funcionais, que exercem efeito específico sobre a dieta devido a sua constituição química, tem sido prioridade de alguns consumidores (Foligné, Daniel \& Pot, 2013; Bezerra, Araújo, Santos \& Correia, 2015).

Uma dieta rica em alimentos funcionais promove o bem-estar, disposição e energia, contribuindo para melhoria da qualidade de vida dos indivíduos (Klein \& Fassina, 2015). A introdução destes na alimentação pode ser favorável para desenvolver uma microbiota intestinal saudável. Os microrganismos presentes na microbiota estão diretamente envolvidos no sistema imune e regulam as respostas às bactérias patógenas, prevenindo o desenvolvimento de novas doenças, contribuindo para a manutenção e promoção da saúde.

A composição da microbiota é obtida no nascimento e sua composição final é adquirida por volta dos dois anos de idade, acompanhando o ser humano até o final da sua vida, podendo ocorrer modificações por razões adversas, intrínsecas ou extrínsecas ao hospedeiro, a saber: uso de antibióticos, de prebióticos e probióticos na alimentação, fatores genéticos, idade, estresse, entre outros (Zhang et al., 2015). Por outro lado, o consumo desses alimentos funcionais modifica e proporciona benefícios a saúde do indivíduo (Barbosa, Costa, Alfenas, Paula \& Minin, 2010; Paixão \& Dos Santos, 2016). O desequilíbrio ocasionado por esses fatores reflete na modificação da microbiota, com diminuição de bactérias benéficas e aumento de patógenos, caracterizando um quadro de disbiose (Zhang et al., 2015). Esse desequilíbrio da microbiota mucosa resultar da desregulação imune na mucosa do intestino, sendo associada a um número de doenças inflamatórias e imunomediadas. Obter uma homeostase adequada durante o momento de colonização do trato gastrointestinal (TGI) é um dos principais elementos para a modulação do sistema imune adequada e indução da tolerância imunológica. O não funcionamento desse sistema é a grande causa de doenças autoimunes ou atópicas (Satokari et al., 2014; Francino, 2014).

\section{Metodologia}

Trata-se de uma pesquisa de revisão narrativa, de caráter descritivo, qualitativo. As bases de dados utilizados para pesquisa foram: Scientific Electronic Library Online (SciELO); Literatura Latino-Americana e do Caribe em Ciências da Saúde - LILACS, Biblioteca Virtual de Saúde (BVS), Google acadêmico, Medline e Pubmed de janeiro a dezembro de 2020.

Determinaram-se para a execução da pesquisa os descritores específicos e controlados, disponíveis: Probióticos/Probiotics, Probióticos e diabetes/Probiotics and diabetes e Probióticos e câncer/Probiotics and cancer. Durante a etapa de busca, estabeleceram-se os seguintes critérios de inclusão: artigos completos publicados na íntegra, 
disponíveis nos idiomas inglês, espanhol e português atribuindo-se o recorte temporal nos últimos 20 anos (2000-2020). Inicialmente encontrado 12.000 artigos com o descritor probióticos, ao aplicar o recorte temporal e demais descritores, foi obtido o resultado final de 210 artigos.

Por conseguinte, a análise dos estudos foi feita por meio de uma avaliação criteriosa dos achados na plataforma, com a leitura do título e resumo simples, sondando assim os artigos que realmente se integravam aos critérios de inclusão, metodologia e temática, excluindo-se os fora de contexto, e posteriormente, uma confirmação da análise pela leitura do texto completo (Moher, Liberati, Tetzlaff \& Altman, 2015).

\section{Resultados e Discussão}

\subsection{Probióticos: definições e principais microrganismos utilizados}

A Organização Mundial da Saúde (OMS) define probióticos como microrganismos vivos que, administrados em quantidades adequadas, são capazes de promover benefícios ao hospedeiro a partir da supressão de patógenos endógenos e exógenos, beneficiando a resposta imune (Ganguly et al., 2011).

Atualmente, os probióticos têm sido amplamente estudados e propagados, com fácil aceitação na alimentação para auxiliar na regulação da microbiota intestinal (Domingo, 2017). Seus efeitos consistem em estimular a proteção contra bactérias levando a danos, como diminuição da permeabilidade intestinal e desempenho inadequado da atividade do sistema imunológico (Paixão \& Dos Santos, 2016).

Os probióticos está sendo muito pesquisado, tendo os principais vetores para promoção de ação nos diferentes microrganismos endógenos humanos, visando ao desenvolvimento do processo de saúde no hospedeiro (Zhu, Sunagawa, Mende, \& Bork, 2015; American, 2018). Esses agentes, que podem estar presentes em alimentos e também em alguns produtos específicos, têm sido utilizados em benefício da saúde e, em alguns casos, no tratamento adjuvante de algumas doenças. As principais cepas utilizadas são dos gêneros Lactobacillus e Bifidobacterium. O histórico de utilização bem como sua regulamentação e seus ganhos sistêmicos encorajaram o uso dessa terapia para diversas doenças associadas ou causadas por bactérias (Cambiaghi, Sant'Ana, Rezende, Greghi \& Damante, 2013).

Os probióticos principais são: o Lactobacillus acidophilus, Lactobacillus casei, Lactobacillus plantarum, Lactobacillus reuteri, Lactobacillus rhamnosus, Lactobacillus paracasei, Bifidobacterium bifidum, Bifidobacterium breve, Bifidobacterium infantis, Bifidobacterium lactis, Bifidobacterium longum, Bifidobacterium adolescentis, Saccharomyces bourlardii, Propionibacterium freudenreichii. São considerados também Escherichia, Enterococcus e Bacillus e o fungo Saccharomyces oulardii. No entanto, apesar de serem benéficos ao organismo e de serem frequentemente adicionados à alimentação infantil, os Lactobacillus bulgaricus e Streptococcus thermophilus (Morais \& Jacob, 2006).

Os probióticos estão sujeitos aos regulamentos contidos na lei geral dos alimentos, segundo a qual eles devem ser seguros para a saúde humana e animal. Nos Estados Unidos da América os microrganismos usados para consumo devem ter o status GRAS (Geralmente Considerado Seguro), regulamentado pelo FDA (Comida e Administração de Drogas). Na Europa, a EFSA (Autoridade Europeia para a Segurança dos Alimentos) introduziu o termo QPS (Presunção Qualificada de Segurança). O conceito QPS envolve alguns critérios adicionais da avaliação de segurança de suplementos bacterianos, incluindo o histórico de uso seguro e a ausência do risco de resistência adquirida a antibióticos (Anadón, Martínez-Larranaga, \& Martínez, 2006; Gaggia, Mattarelli \& Biavati, 2010).

A Tabela 1 apresenta os microrganismos probióticos contidos em produtos farmacêuticos e como aditivos alimentares. 
Tabela 1. Microrganismos probióticos usados na nutrição humana.

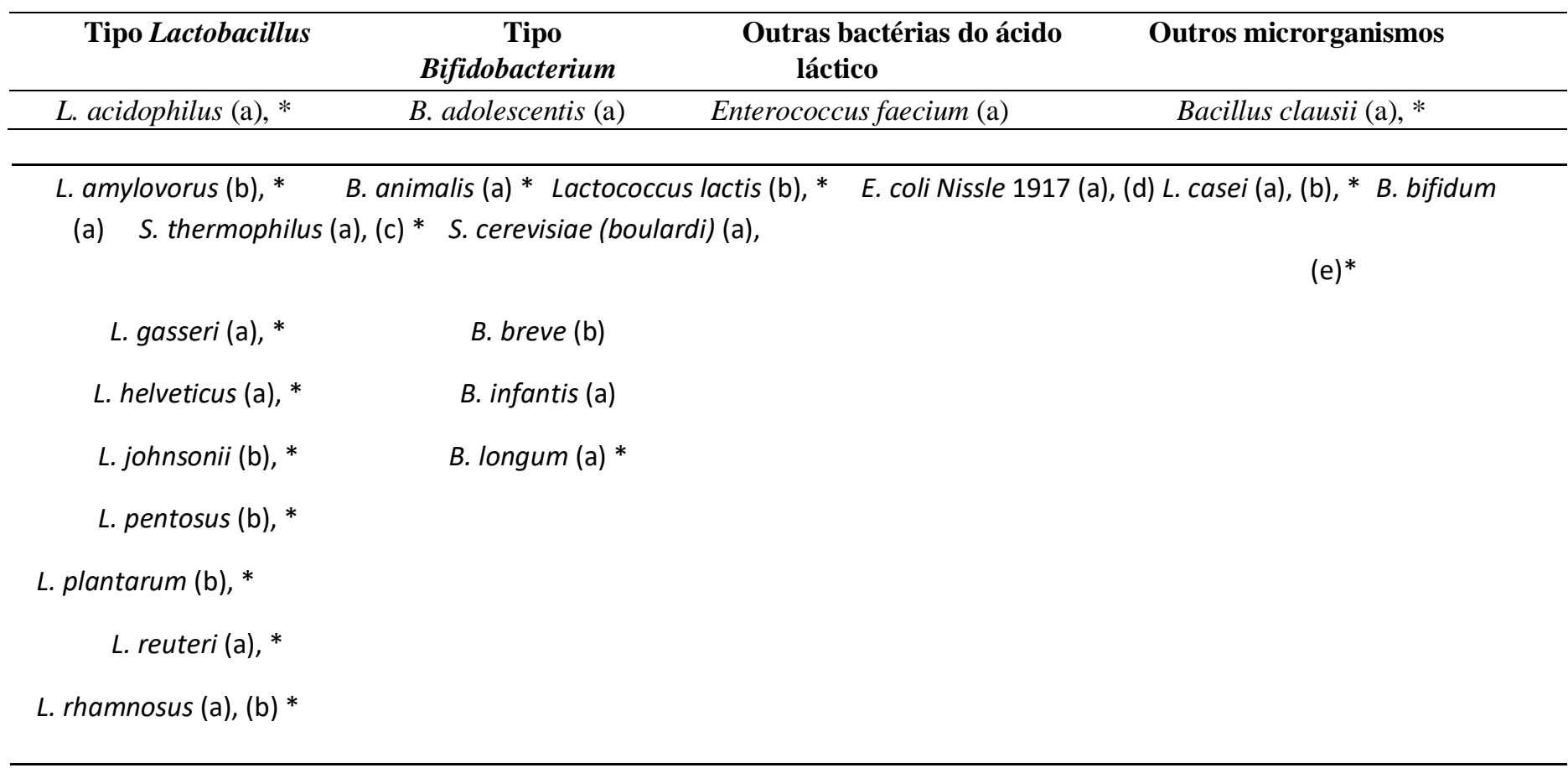

LEGENDA:(a) Principalmente como produtos farmacêuticos; (b) principalmente como aditivos alimentares; (c) Streptococcus; (d) Escherichia; (e) Saccharomyces; * Microrganismos QPS (Presunção Qualificada de Segurança).

FONTE: AESA, 2013; AESA 2017.

\subsection{Epidemiologia do câncer e diabetes mellitus}

No Brasil, as doenças crônicas não transmissíveis (DCNT) foram responsáveis por 73\% dos óbitos em 2016 (OMS, 2017). De acordo com o Plano de Ações Estratégicas para o Enfrentamento das DCNT no país de 2011 a 2022 , a hipertensão e a diabetes constituem causas centrais de morbimortalidade no país, com elevadas repercussões sociais e econômicas (Ministério da Saúde, 2011).

O câncer e o diabetes são doenças crônicas, consideradas pela Organização Mundial da Saúde (OMS) como problemas prioritários de saúde pública global. A Agência Internacional de Pesquisa sobre o Câncer (IARC) estimou a incidência global de câncer em 2018 em 18,1 milhões, em todas as idades e sexos combinados: 9,5 milhões em homens e 8,6 milhões em mulheres, acompanhados por 9,6 milhões de mortes: 5,4 milhões em homens e 4,2 milhões em mulheres. As regiões do mundo que mais contribuíram para o aumento desses números são, pela ordem: Ásia (48,4\%), Europa $(23,4 \%)$ e Américas (21\%) para incidência e Ásia (57,3\%), Europa (20,3\%) e Américas / Caribe (14,4\%) para mortalidade (Bray et al., 2018).

As neoplasias malignas estão em segundo lugar na causa de mortalidade no Brasil (Torres-Pereira, AngelinDias, Melo, Lemos Jr. \& Oliveira, 2012). Em 2030 o câncer deverá ser a principal causa de morte no país, aumentando os custos de novas tecnologias para seu tratamento, levando a um sério desafio à saúde pública (Barbosa, Costa, Bernal \& Souza, 2016).

O câncer é atualmente classificado como a segunda causa de morte em todo o mundo, bem como a primeira ou segunda causa de morte prematura em 91 de 172 países, e como a terceira ou quarta causa em 22 outros países (Fitzmaurice, 2017). O risco cumulativo e a probabilidade de morrer prematuramente de câncer para ambos os sexos são 21,4 e 17,7\%, respectivamente; ou seja, em 2018, 1 em cada 5 homens e 1 em cada 6 mulheres sofriam de algum tipo de câncer, e 1 em cada 8 homens e 1 em cada 10 mulheres morriam dessa doença (Bray et al., 2018). 
A prevalência de diabetes no mundo em 2017 foi de 8,8\% na faixa etária de 20 a 79 anos, correspondendo a 424,9 milhões de pessoas, com estimativa de 9,9\% para 2045, com 628,6 milhões de diabéticos. No Brasil, em 2017, diagnosticou 12,5 milhões de pessoas com diabetes mellitus, com uma prevalência de 8 a 9\%, ocupando o quinto lugar no mundo. Estima-se que existem 46\% (5,7 milhões) de pessoas diabéticas não diagnosticadas, na faixa etária de 20 a 79 anos, ocupando o quarto lugar entre os 10 países no mundo. A mortalidade mundial atingiu 4 milhões de pessoas, sendo que metade desses óbitos foram no Brasil (IDF, 2017).

As complicações agudas e crônicas do diabetes causam alta morbimortalidade, acarretando altos custos para os sistemas de saúde. Gastos relacionados ao diabetes mundialmente, em 2010, foram estimados em 11,6\% do total dos gastos com atenção em saúde (IDF, 2012).

A utilização da epidemiologia para organização e monitoramento das condições de saúde da população ajuda no controle de incidências e chama atenção dos órgãos de saúde pública para prevenção e tratamentos das doenças.

\subsection{Uso de probióticos no tratamento do câncer e diabetes}

Alguns pesquisadores acreditam que os microrganismos probióticos seriam a proteção do hospedeiro contra atividades carcinogênicas por meio de três mecanismos principais: a inibição das bactérias responsáveis por converter substâncias pré-carcinogênicas em carcinogênicas; inibição direta de formação de células tumorais; e a capacidade de algumas bactérias da microbiota intestinal de ligação e/ou inativação carcinogênica (Damião, Leite, Lordello \& Sipahi, 2009).

O consumo de probióticos protege contra o desenvolvimento do câncer, pode ser atribuído a várias explicações biológicas gerais: melhorar o sistema imunológico do hospedeiro, modular o estresse oxidativo e a inflamação ou manter as populações bacterianas saudáveis, de forma que elas superem/suprimam as bactérias que produzem agentes cancerígenos, no entanto, mais especificamente (Kumar et al., 2010; Catana, Cristea, Miron \& Neagoe, 2011; Gata et al., 2017).

A administração de alguns prebióticos e probióticos podem melhorar as alterações metabólicas associadas à obesidade e diabetes, como resistência à insulina, hiperglicemia, inflamação, dislipidemia ou DHGNA em animais (Bashiardes, Shapiro, Rozin, Shibolet \& Elinav, 2016). Por exemplo, a administração de probióticos, pode contribuir para uma melhora modesta no controle da glicose no sangue (Festi et al., 2014). Além disso, outros pesquisadores demonstraram que o uso de prebióticos, probióticos e simbióticos está associado a pequenas melhorias no controle lipídico (Sáez-Lara, Robles-Sanchez, Ruiz-Ojeda, Plaza-Diaz \& Gil, 2016).

Como microrganismos vivos não patogênicos, os probióticos são benéficos para os anfitriões, eles também melhoram o sistema imunológico em muitos órgãos através da mmaturação de linfócitos (Prithy \& Yoshinori, 2012), também exibem efeitos antimicrobianos e podem melhorar dislipidemia, diabetes tipo 2 e colesterol sérico esterol (Singh et al., 2012). Entre esses efeitos, a propriedade antidiabética se tornou um importante foco de pesquisa. Por exemplo a progressão do diabetes mellitus pode ser efetivamente evitada ou atrasado usando vários probióticos (Hung, Tseng \& Pan, 2016).

Estudos recentes provaram que bactérias ácidas e probióticos têm destaque em efeitos positivos em humanos ou animais com diabetes tipo1 e 2. Nos modelos animais com diabetes tipo 2, foi investigado e confirmou a capacidade de Lactobacilos para reduzir o risco de desenvolvimento (Andersson et al., 2010). Como suplementos probióticos, Lactobacillus plantarum DSM 15313 e L gasseri BNR17 são estudados em camundongos modelos induzidos a obesidade e diabetes da dieta lipídica alta, apresentaram efeito anti-diabetes, diminuindo o nível de glicose no sangue e melhora a tolerância sem influência de lipídios e níveis de insulina (Andersson et al., 2010; Yun, Park \& Kang, 2009). 
Um crescente corpo de evidências sugere que associações favoráveis são existentes entre o consumo de probióticos e o perfil metabólico entre diabéticos (Kasińska, \& Drzewoski, 2015). No entanto, os mecanismos potenciais subjacentes aos efeitos dos probióticos nos parâmetros relacionados à glicemia não são totalmente compreendidos. Um dos principais mecanismos postulados pode envolver aumento do peptídeo 1 semelhante ao glucagon (GLP1) pelas secreções e células $L$ enteroendócrinas para melhorar os carboidratos no metabolismo, diminuem a glicotoxicidade e aumentam a sensibilidade à insulina nas células alvo (Tremaroli \& Bäckhed, 2012). Outros mecanismos propostos para explicar a ação dos probióticos no diabetes referem-se a anti-inflamatórios, antioxidantes e efeitos imunomoduladores e alteração da expressão de alguns genes envolvidos no diabetes (Kim \& Sears, 2010; Balakumar et al., 2016).

Em um estudo in vitro e in vivo sobre a cepa probiótica Lactobacillus acidophilus SJLH001 (La-SJLH001) constatou-se que a cepa possui diferentes propriedades probióticas. La-SJLH001 atenuado hiperglicemia e hipercolesterolemia induzidas por dieta rica em gorduras. Resultados mostraram a melhoria da homeostase da glicose e colesterol metabolismo em camundongos obesos induzidos, essas descobertas são importantes para a uma aplicação mais bem-sucedida de probióticos em prevenções e inclusão em alimentos funcionais no futuro (Sun et al., 2019).

\section{Conclusão}

Diante do exposto, a utilização de probióticos tem um papel benéfico no tratamento de doenças crônicas não transmissíveis como câncer e diabetes. No câncer, as bactérias probióticas reduzem os efeitos mutagênicos e genotóxicos, nosso trabalho também demonstra a escassez de artigos relacionados, demonstrando a necessidade de mais pesquisas científicas.

\section{Referências}

American Gut (2018) http://americangut. org/.

Anadón, A., Martínez-Larranaga, M., \& Martínez, M. (2006). Probióticos para nutrição animal na Europa União. Regulamentação e avaliação de segurança. Regul. Toxicol. Pharmacol. 45, 91-95.

Andersson, U., Branning, C., Ahrne, S., Molin, G., Alenfall, J., Onning, G., Nyman, M., \& Holm, C. (2010). Probióticos diminuem a glicose plasmática no camundongo C57BL/6J alimentado com alto teor de gordura. Benef. Microbes, 1: 189-196.

ANVISA - Agência Nacional de Vigilância Sanitária. (1999). Conselho Nacional de Segurança Alimentar e Nutricional - Consea. https://issuu.com/informecaisan/docs/guiaalimentarpopulacaobrasileira_20

Autoridade Europeia para a Segurança dos Alimentos (AESA). (2013). O relatório de síntese da União Europeia sobre tendências e fontes dezoonoses, agentes zoonóticos e surtos de origem alimentar em 2011. EFSA J. 3129, 1-250.

Autoridade Europeia para a Segurança dos Alimentos (AESA). (2017). Parecer científico sobre a atualização da lista de QPS recomendados agentes biológicos adicionados intencionalmente a alimentos ou rações, conforme notificado à EFSA (atualização de 2017). EFSA J. 15,1-177.

Balakumar, M., Prabhu, D., Sathishkumar, C., Prabu, P., Rokana, N., Kumar, R., Raghavan, S., Soundarajan, A., Grover, S., Batish, V., Mohan, V., \& Balasubramanyam, M. (2016). Melhoria na tolerância à glicose e sensibilidade à insulina por cepas probióticas de origem intestinal indiana em camundongos C57BL / 6J alimentados com dieta rica em gordura. Eur J Nutr.

Barbosa, I., Costa, I., Bernal, M., \& Souza, D. (2016). Tendência das taxas de mortalidade pelas dez principais causas de óbitos por câncer no Brasil, 1996-2012. Rev. Ciênc. Plur. 2 (1): 3-16.

Barbosa, K., Costa, N., Alfenas. R., Paula. S., \& Minin, V. (2010). Estresse oxidativo: conceito, implicações e fatores moduladores. Revista de Nutrição, 23(4), 629-643.

Bashiardes, S., Shapiro, H., Rozin, S., Shibolet, O., \& Elinav, E. (2016). Fígado gordo não alcoólico e microbiota intestinal. Mol Metab. 5 (9): 782794.

Bezerra, M., Araujo, A., Santos. K., \& Correia, R. (2015). Iogurte congelado caprino produzido com polpa de fruta de jambolã fresca e seca por atomização (Eugenia jambolana Lam) e Bifidobacterium animal subespécie lactis BI-07. Ciência Alimentar e Tecnologia. 1, 16.

Brasil. (2013). Ministério da Saúde. Secretaria de Atenção à Saúde. Departamento de Atenção Básica. Políticatolo Nacional de Alimentação e Nutrição. 
Bray, F., Ferlay, J., Soerjomataram, I., Siegel, R., Torre, L., \& Jemal, A. (2018). Estatísticas globais de câncer de 2018: estimativas GLOBOCAN de incidência e mortalidade mundial para 36 cânceres em 185 países. CA Cancer J Clin. 68 (6): 394-424.

Cambiaghi, L., Sant'Ana, A., Rezende, M., Greghi, S., \& Damante, C. (2013). Os probióticos na odontologia: história, conceitos e aplicações na periodontia. Perionews, 7(1):18-24.

Catana, C., Cristea, V., Miron, N., \& Neagoe, I. (2011). A interleucina-17 é um biomarcador pró-aterogênico? Roma. Arco. Microbiol. Immunol. 70 , $124-128$.

Damião, A., Leite, A., Lordello, M., \& Sipahi, A. (2009). Probióticos no: Waitzberg LD. Nutrição Oral, Enteral e Parenteral na Prática Clínica. (4a ed.), Editora Atheneu.

Domingo, J. (2017). Revisão do papel dos probióticos nas doenças gastrointestinais em adultos. Gastroenterologia e Hepatologia, 40: $417-429$.

Federação Internacional de Diabetes (IDF). (2012). Atualização do atlas de diabetes de 2012:

Fichas técnicas regionais e nacionais. http://www.idf.org/diabetes-atlas-update-2012-reginal-country-factsheets

$\begin{array}{llllllll}\text { Federação Internacional } & \text { de } & \text { Diabetes } & \text { (IDF). } & \text { (2017). } & \text { IDF } & \text { Diabetes }\end{array}$ [https://diabetesatlas.org/upload/resources/previous/files/8/IDF_DA_8e-EN-final.pdf

Festi, D., Schiumerini, R., Eusebi, L., Marasco, G., Taddia, M., \& Colecchia, A. (2014). Microbiota intestinal e síndrome metabólica. Mundo J. Gastroenterol. 20 (43): 16079-16094.

Fitzmaurice, C., Allen, C., Barber, R., Barregard, L., Bhutta, Z., \& Brenner, H. (2017). Incidência global, regional e nacional de câncer, mortalidade, anos de vida perdidos, anos vividos com deficiência e anos de vida ajustados por deficiência para 32 grupos de câncer, 1990 a 2015 : Uma análise sistemática para o estudo Global Burden of Disease. JAMA Oncol. 3 (4): 524-548.

Foligné, B., Daniel, C., \& Pot, B. (2013). Probióticos da pesquisa ao mercado: as possibilidades, riscos e desafios. Opinião Atual em Microbiologia, $16,284-292$.

Francino, M. (2014). Desenvolvimento precoce da microbiota intestinal e saúde imune. Patógenos, Basileia, 3(3), 769-790.

Gaggia, F., Mattarelli, P., \& Biavati, B. (2010). Probióticos e prebióticos na alimentação de animais para produção segura de alimentos. Int. J. Food Microbiol. 141, S15-S28.

Ganguly, N., Bhattacharya, S., Sesikeran, B., Nair, G., Ramakrishna, B., Sachdev, H., Batish, V., Kanagasabapathy, A., Muthus wamy, V., Kathuria, S., Katoch, V., Satyanarayana, K., Toteja, G., Rahi, M., Rao, S., Bhan, M., Kapur, R., \& Hemalatha, R. (2011). ICMR-DBT Diretrizes para avaliação de probióticos em alimentos. Indian J Med Res. 134: 22-5.

Gata, V., Lisencu, C., Vlad, C., Piciu, D., Irimie, A., \& Achimas-Cadariu, P. (2017). Tumor infiltrando linfócitos como fator prognóstico no melanoma maligno. Revisão da literatura. J. BUON, 22, 592-598.

Gavanski, D., Baratto, I., \& Gatti, R. (2015). Avaliação do hábito intestinal e ingestão de fibras alimentares em uma população de idosos. Revista brasileira de obesidade, nutrição e emagrecimento, 9(49), 3-11.

Hung, S., Tseng, W., \& Pan, T. (2016). Lactobacillus paracasei subsp. paracasei NTU 101 melhora a tolerância à glicose diminuída induzida por uma dieta rica em gordura e frutose em ratos Sprague-Dawley. J. Funct. Foods, 24, 472-481.

Kasińska, M., \& Drzewoski, J. (2015). Efetividade dos probióticos na diabetes tipo 2: uma meta-análise. Pol Arch Med Wewn. 125 (11): 803 -13.

Kim, J., \& Sears, D. (2010). TLR4 e resistência à insulina. Gastroenterol Res Pract.20. pii: 212563.

Klein, S., \& Fassina, P. (2015). Relação entre o consumo de alimentos funcionais e alterações fisiológicas em praticasountes de atividade física. Caderno Pedagógico. Lajeado, v.12, n.1, p.22-35.

Kumar, M., Kumar, A., Nagpal, R., Mohania, D., Behare, P., Verma, V., Kumar, P., Poddar, D., \& Aggarwal, P., (2010) Atributos de probióticos que previnem o câncer: uma atualização. Int. J. Food Sci. Nutr. 61, 473-496.

Kuper, H., Adami, H., \& Trichopoulos, D. (2000). Infecções como uma das principais causas evitáveis de câncer humano. J. Intern. Med. 248 , 171183.

Martins, D., Walder, B., \& Rubiatti, A. (2010). Educação nutricional: atuando na formação de hábitos alimentares saudáveis de crianças em idade escolar. Ver Simbiologias. 3(4):86102.

Ministério da Saúde (BR), Secretaria de Vigilância em Saúde, Departamento de Análise de Situação de Saúde. (2011). Plano de ações estratégicas para o enfrentamento das doenças crônicas não transmissíveis (DCNT) no Brasil: 2011-2022. Brasília (DF).

Moher, D., Liberati, A., Tetzlaff. J., \& Altman, D. (2015). Principais itens para relatar Revisões Sistemáticas e Meta-análises: A recomendação PRISMA. Rev Epidemiol Serv Saúde, 24 (2), 335-342.

Morais, M., \& Jacob, C. (2006). O papel dos probióticos e prebióticos na prática pediátrica. J Pediatr. 82 (5): S189-S197.

Naderi, A., Kasra - Kermanshahi, R., Gharavi, S., Fooladi, A., Alitappeh, M., \& Saffarian, P. (2014). Estudo dos efeitos antagônicos de cepas de Lactobacillus como probióticos em bactérias multirresistentes (MDR) isoladas de infecções do trato urinário (UTIs). Jornal Iraniano de Ciências Médicas Básicas. 17 (3): 201. 
Research, Society and Development, v. 10, n. 5, e41910514932, 2021

(CC BY 4.0) | ISSN 2525-3409 | DOI: http://dx.doi.org/10.33448/rsd-v10i5.14932

Organização Mundial de Saúde. (2017). Monitor de progresso de doenças não transmissíveis. Genebra: OMS.

Paixão, L., \& Dos Santos, F. (2016). Colonização da microbiota intestinal e sua influência na saúde do hospedeiro. Universitas: Ciências da Saúde, 14(1), 85-96.

Prithy, R., \& Yoshinori, M. (2012). Avanços recentes no papel de probióticos na inflamação humana e na saúde intestinal. J. Agric.Food Chem, 60, 8249-8256.

Sáez-Lara, M., Robles-Sanchez, C., Ruiz-Ojeda, F., Plaza-Diaz, J., \& Gil, A. (2016). Efeitos de probióticos e simbióticos na obesidade, síndrome de resistência à insulina, diabetes tipo 2 e doença hepática gordurosa não alcoólica: uma revisão de ensaios clínicos em humanos . Int J Mol. Sci. 17 (6).

Satokari, R., Fuentes, S., Mattila, E., Jalanka, J., M. de Vos, W., \& Arkkila, P. (2014). Transplante fecal Tratamento da colite não-infecciosa induzida por antibióticos e acompanhamento da microbiota a longo prazo. Relatos de Casos em Medicina, 913867, 1-7.

Simon, O. (2005). Microrganismos como aditivos para a alimentação animal - Probióticos. Adv. Porco Prod. 16, $161-167$.

Singh, V., Raheja, G., Borthakur, A., Kumar, A., Gill, R., Alakkam, A., Malakooti, J., \& Dudejaz, P. (2012). Lactobacillus acidophilus regula positivamente a expressão e função intestinal de NHE3, alt. J. Physiol: Gastrointest. Physiol of the liver. 303, G1393-G1401.

Sudhakar Reddy, R., Ramesh, T., Rajesh Singh, T., Vijayalaxmi, N., \& Lavanya, R. (2011). Bactérias na saúde bucal - probióticos e prebióticos uma revisão. Jornal Internacional de Pesquisa Biológica e Médica. 2 (4): 1226-1233.

Sun, Q., Yong Zhang, Y., Li, Z., Yan, H., Lib, J., \& Wan, X. (2019). Análise de mecanismo de glicose melhorada homeostase e metabolismo do colesterol em ratos obesos induzidos com alto teor de gordura tratados com La-SJLH001 via transcriptômica e culturômica. Food Funct. $10,3556$.

Toloni, M., Longo-Silva, G., Goulart, R \& Taddei, J. (2011). Introdução de alimentos industrializados e de alimentos de uso tradicional na dieta de crianças de creches públicas no município de São Paulo. Revista de Nutrição de Campinas, 24(1), 61-70.

Torres-Pereira, C., Angelin-Dias, A., Melo, N., Lemos Jr., C., \& Oliveira, E. (2012). Abordagem do câncer de boca: uma estratégia para os níveis primário e secundário de atenção em saúde. Cad Saúde Pública, 28 Supl:S30-9.

Tremaroli, V., \& Bäckhed, F. (2012). Interações funcionais entre a microbiota intestinal e o metabolismo do hospedeiro. Natureza. 489 (7415): $242-9$.

Yun, S., Park, H., \& Kang, J. (2009). Efeito de Lactobacillus gasseri BNR17 nos níveis sanguíneos de glicose e peso corporal em um rato modelo de diabetes tipo 2. J. Appl Microbiol. 107: 1681-1686.

Zhang, Y., Li, S., Gan, R., Zhou, T., Xu, D., \& Li, H. (2015). Impactos das bactérias intestinais na saúde e doenças humanas. Revista Internacional de Ciências Moleculares. 16(4), 7493-7519.

Zhu, A., Sunagawa, S., Mende, D., \& Bork, P. (2015). Diferenças interindividuais no conteúdo gênico de espécies bacterianas do intestino humano. Genoma Biol. 16:82. 